\title{
STIGMA TOWARD MENTAL ILLNESS: LINGUISTIC VALIDATION OF THE ATTRIBUTION QUESTIONNAIRE-27 (AQ-27)
}

\author{
B.N. Saguem ${ }^{1}$, A. Braham¹, M. Gharmoul1 ${ }^{1}$, J. Nakhli1, S. Ben Nasr ${ }^{1}$. \\ ${ }^{1}$ Farhat Hached Hospital, Psychiatry, Sousse, Tunisia.
}

\section{Background and Aims:}

- Stigma often emerges in the context of mental disorders, mainly schizophrenia. It significantly restrains the life opportunities of these individuals.

- This study aimed at performing a linguistic validation of the Attribution Questionnaire-27 (AQ-27).

\section{Materials and Methods:}

- The $\mathrm{AQ}-27$ is a self-administered measure, designed to explain attitudes, affect, and behavioral intentions related to a hypothetical 30-year-old single man suffering from schizophrenia [1].

- Individuals who complete the measure are asked to rate how much they agree with each statement made about the hypothetical man named "Harry" on a Likert scale from 1 (not at all) to 9 (very much) [1].

- Two native Arabic speakers, bilingual in English, independently translated the original measures into Arabic. A collaborative pooled version of the questionnaires was then obtained from the two translations.

- The pooled version was back-translated into English by a professional translator.

- A draft Arabic version of the battery was obtained from the comparaison between the original questionnaires and the back-translation and was tested on 30 Arabic-speaking médical students.

- We discussed with the students about the existence of other wording that enables the meaning of statements to be expressed clearly.

\section{Results:}

- The test of the different 27 items allowed the detection of two typing errors. It also highlighted mistranslating two different terms to one term that doesn't distinguish between them in term of meaning: "Scared " and « Frightened " in items 19 and 24, respectively.

- Comments were reported on the wording of certain items in the questionnaire. These comments referred to the problems related to the grammatical and syntactical construction of certain expressions. The translation from English into Tunisian dialect is different from our dialect.

\section{Discussion:}

- We proposed an Arabic version of the AQ-27, an operationalized instrument designed to explore stigma in psychiatry. This is a first step for reliable measures that assess stigma in Arabic countries.

- The AQ-27 was translated using the standard translation/ back-translation technique along with a test of the target language version (Arabic version) with monolingual subjects. Back-translation is the most highly recommended technique for translation in cross-cultural research [2]. Brislin et al. (1973) recommended one or more of the following techniques: back-translation; bilingual techniques; committee approach and pretest [3].

- A key benefit of conducting a back-translation is that this allows comparison of the original source language version with the version which was back-translated into the source language. Through this process, comparison between source and target language versions by bilingual judge(s), is made, in order that discrepancies of translation can be identified and corrected.

- Despite its benefits, back-translation technique does not solve all the problems of translation, and the existence of discrepancy between the two original and retro-translated versions was inevitable in our study.

- In back-translation, modification of words and concepts that have no clear equivalence in the other language is allowed. This is particularly useful when one version has ideas and words which, in the other language, seem socially insensitive or can be expressed only with difficulty [4]. The key to achieving semantic equivalence is to retain the same meaning of each item after translation into the language of each culture [5]. This technique also helps to achieve conceptual equivalence.

- Combining back-translation with testing of the target language version among monolingual subjects is necessary to test the clarity and appropriateness of the target language version. Ideally, this should involve comprehensive psychometric evaluation to assess reliability and validity, as would be performed with any newlydeveloped instrument [2].

\section{References:}

[1] Corrigan, P., Markowitz, F., Watson, A., Rowan, D., Kubiak, M., 2003. An attribution model of public discrimination towards persons with mental illness. Journal of Health and Social Behavio 44,162-179. [2] Ma 1 , Jurn. Transtation procedures for the cross cultural use of meaneme Fransation procedures for the cross cultural use of measurement instruments. Educational Wintrob, R, Richman, JA, Birz, S, 1988. Developing instum Wintrob, R., Richman, J.A., Birz, S., 1988. Developing instruments for cross- cultural psychiatric esearch. Journal of Nervous and Mental Disease 176, 257-263. 